\title{
New Position Algorithms for the 3-D CZT Drift Detector
}

\section{Budtz-Jørgensen, Carl; Kuvvetli, Irfan}

\section{Published in:}

IEEE Transactions on Nuclear Science

Link to article, DOI:

10.1109/TNS.2017.2696574

Publication date:

2017

Document Version

Peer reviewed version

Link back to DTU Orbit

Citation (APA):

Budtz-Jørgensen, C., \& Kuvvetli, I. (2017). New Position Algorithms for the 3-D CZT Drift Detector. IEEE Transactions on Nuclear Science, 64(6), 1611-1618. https://doi.org/10.1109/TNS.2017.2696574

\section{General rights}

Copyright and moral rights for the publications made accessible in the public portal are retained by the authors and/or other copyright owners and it is a condition of accessing publications that users recognise and abide by the legal requirements associated with these rights.

- Users may download and print one copy of any publication from the public portal for the purpose of private study or research.

- You may not further distribute the material or use it for any profit-making activity or commercial gain

- You may freely distribute the URL identifying the publication in the public portal

If you believe that this document breaches copyright please contact us providing details, and we will remove access to the work immediately and investigate your claim 


\title{
New Position Algorithms for the 3D CZT Drift Detector
}

\author{
C. Budtz-Jørgensen and I. Kuvvetli
}

\begin{abstract}
The 3D position sensitive CZT detector for high energy astrophysics developed at DTU has been investigated with a digitizer readout system. The 3D CZT detector is based on the CZT Drift Strip detector principle and was fabricated using a REDLEN CZT crystal $(20 \mathrm{~mm} \times 20 \mathrm{~mm} \times 5 \mathrm{~mm})$. The detector contain 12 drift cells, each comprising one collecting anode strip with 4 drift strips, biased such that the electrons are focused and collected by the anode strips. 3D position determination is achieved using the anode strip signals, the drift strip signals and the signals from 10 cathode strips.
\end{abstract}

For the characterisation work, we used a DAQ system with a 16 channels $250 \mathrm{MHz}$ 14bit digitizer, SIS3316. It allowed us to analyse the pulse shapes of the signals from 4 detector cells at a time.

The 3D CZT setup was characterized with a finely collimated radioactive source of ${ }^{137} \mathrm{Cs}$ at $662 \mathrm{keV}$. The analysis required development of novel position determination algorithms which are the subject of this paper.

Using the digitizer readout we demonstrate improved position determination compared to the previous read out system based on analog electronics. Position resolutions of 0.4 mm FWHM in the $\mathrm{X}$-, $\mathrm{Y}$ - and $\mathrm{Z}$-directions were achieved and the energy resolution was $7.2 \mathrm{keV}$ FWHM at $662 \mathrm{keV}$.

The timing information allows identification of multiple interaction events within one detector cell, e.g. Compton scattering followed by photoelectric absorption.

These characteristics are very important for a high energy spectral-imager suitable for use in advanced Compton telescopes, or as focal detector for new hard $X$-ray and soft gamma-ray focusing telescopes or in polarimeter instrumentation. CZT detectors are attractive for these applications since they offer relatively high quantum efficiency. From a technical point of view it is advantageous that their cooling requirements are modest.

Index Terms-3D CZT detectors, Compton camera, CZT Drift Strip Detectors, Digitized pulse shape analysis, High Energy Astrophysics, Position Algorithms.

\section{INTRODUCTION}

At DTU Space, we have developed a high resolution three dimensional (3D), position sensitive CZT detector for high energy astronomy [1] and [2]. The design of the 3D CZT detector is based on the CZT Drift Strip detector principle [3]. IMEM-CNR (Parma, Italy) fabricated 4 prototype detectors to

I. Kuvvetli is a senior scientist at the Technical University of Denmark, Kgs. Lyngby, DK-2800, Denmark (e-mail: Irfan@ space.dtu.dk).

C. Budtz-Jørgensen is an emeritus at the Technical University of Denmark, Kgs. Lyngby, DK-2800, Denmark (e-mail: Carl@ space.dtu.dk). our specifications. REDLEN CZT crystals (size $20 \mathrm{~mm}$ x 20 $\mathrm{mm} \times 5 \mathrm{~mm}$ ) with high spectral quality, $\mu \tau_{\mathrm{e}}=0.01 \mathrm{~cm}^{2} / \mathrm{V}$, were used for the proto type detectors [2]. Existing electrodes were removed from the REDLEN CZT crystals and reworked following by surface treatment and strip electrode depositions according to our specifications. The detector thickness was in reduced to $4.5 \mathrm{~mm}$ by this process. The $3 \mathrm{D} \mathrm{CZT}$ electrode geometry comprises strip electrodes with $0.4 \mathrm{~mm}$ pitch $(0.15$ $\mathrm{mm}$ width and $0.25 \mathrm{~mm}$ gap) on one side and 10 cathode strips with $2.0 \mathrm{~mm}$ pitch $(1.9 \mathrm{~mm}$ width and $0.1 \mathrm{~mm}$ gap) on the other side.

The 3D prototype detector was configured with 12 drift cells, each comprising one collecting anode strip with 4 drift strips, two on the left and two on the right side of the anode strip. The completed drift cell dimensions are $1.6 \mathrm{~mm} \times 4.5 \mathrm{~mm} \times$ $20 \mathrm{~mm}$. The electrical diagram of the 3D CZT detector is shown in Fig. 1. The drift strips are biased such that the electrons are focused and collected by the anode strips [3].

Fig. 2 shows the electrical layout of a single drift cell. The cathode strips are biased negatively, $\mathrm{HV}=-350 \mathrm{~V}$. A voltage divider supplies the drift strips with a bias supply of $\mathrm{Vi}=\mathrm{Vd}^{*}(\mathrm{i} / 3), \mathrm{i}=1,2$ and $\mathrm{Vd}=-150 \mathrm{~V}$, whereas the anode strips are held at ground potential. The anode and the cathode strips are connected to low noise charge sensitive preamplifiers $(\mathrm{eV}$ 5093), which are fed to the SIS3316 digitizer. The drift strips are AC-coupled together in two groups of left (DL) and right (DR) strips and connected to their respective preamplifier. The 16 channels of the SIS3316 digitizer allow the 4 detector cells (10 cathode strips, 2 drift strip groups and 4 anode strips) to be analyzed simultaneously. The anode strips of the 8 unused detector cells are both DC and AC grounded.

The anode strips provide the energy signals to be analyzed. The weighting potential of the small anode strip ensures that the induced charge of the drifting electrons is nearly proportional to the deposited photon energy. See, the detailed explanation in [3]

The position determination perpendicular to the anodes, in the $\mathrm{X}$-direction, is performed using the signals from the two drift strip groups (DL, DR) and a novel interpolating technique [1]. The position determination through the detector thickness, Ydirection, is made using the depth-sensing technique based on the detector cathode and anode signals. The position determination along the anode strips, Z-direction is made with the help of the 10 cathode strips orthogonal to the anode strips. The detector thus provides 3D position as well as high resolution energy information, and is therefore a well suited candidate e.g. as a focal plane detector for new hard X-ray/soft gamma-ray focusing optics [5], as an advanced Compton telescope detector or a hard X-ray polarimeter [6]. 
The prototype detector was investigated at the European Synchrotron Radiation Facility, Grenoble, in 2013, which provided a fine $50 \times 50 \mu \mathrm{m}^{2}$ collimated $\mathrm{X}$-ray beam covering an energy band up to $700 \mathrm{keV}$, see [2]. The detector employed during these tests used conventional analog readout electronics. Although the tests performed at ESRF demonstrated the basic performance of the detector they also made it clear that this readout system led to a number of challenges:

1. The measured X-positions showed a strong correlation with the Y position of the photon interaction point. This necessitates a complex calibration process, see [2].

2. The Z-position determination becomes rather uncertain for event interactions close to the cathode plane. Here the uncertainty reaches the same value as the pitch of the cathode strips.

3. The analog electronics used at ESRF did not allow that the detector signal waveforms were to be analysed and it was therefore not possible to identify Compton events with two or more interactions in the same detector cell. We therefore decided to equip the detector with a digitizer in order to determine the temporal behaviour of all electrode signals.

The following sections describe the analysis algorithms we developed and the results we obtained.

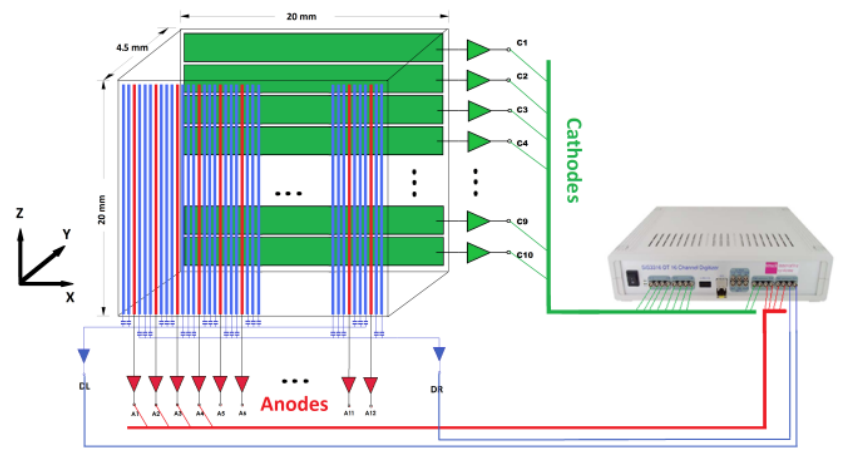

Fig. 1. Electrical diagram of the 3D CZT detector.

\section{3D CZT DETECTOR OPERATIONS}

For the characterisation work, we replaced the conventional DAQ system using pulse-height analysis with a high speed 14bit digitizer, SIS3316 (The Struck Innovative System, $\mathrm{GmbH}$ ), in order to record 4 ns sample data from 16 channels. The electrical diagram of the 3D CZT detector setup is shown in Fig. 1. The 16 channels of the digitizer allowed that 4 detector cells to be connected: 4 anode strips, 2 drift strip groups and 10 cathode strips. The present measurements were performed with a mono energetic $(662 \mathrm{keV}) 1 \mathrm{D}$ slit beam produced with the help of a $10 \mathrm{mCi}{ }^{137} \mathrm{Cs}$ source and a tungsten collimator with an opening of $150 \mu \mathrm{m} \times 40 \mathrm{~mm}$ and a thickness of $60 \mathrm{~mm}$. The detector was mounted close to the collimator $(\sim 20 \mathrm{~mm})$ on a step motor-controlled fixture. Due to the distance of $20 \mathrm{~mm}$ between the collimator opening and the detector surface, the broadening effect of the beam width is estimated to be $225 \mathrm{um}$ in the middle of the detector. The set-up allowed that the detector could be scanned with the 662
$\mathrm{keV}$ slit beam in any detector axis $(\mathrm{X}, \mathrm{Y}, \mathrm{Z})$ with an accuracy of $5 \mu \mathrm{m}$.

\section{A. Single interaction event}

Fig. 2. displays the layout of a single drift cell. Electrons created by the incoming photon are drifted to the anode readout (green) by the electric field provided by the drift electrodes (red). The voltage divider shown to the right provides the required bias voltages. The hole movement can be ignored since $\mu \tau_{\mathrm{e}}>>\mu \tau_{\mathrm{h}}$ for the present CZT material. Details of the CZT drift strip detector are provided in [2], [3].

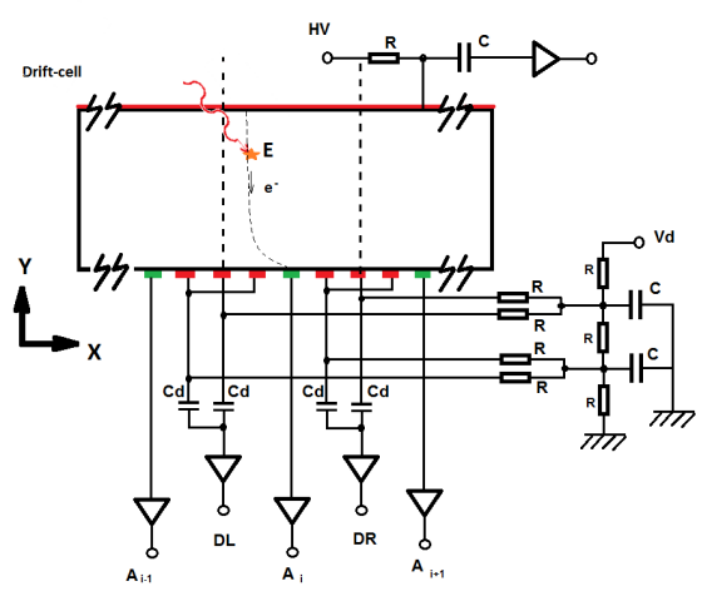

Fig. 2. Electrical diagram of a single drift cell. The drift cell borders are marked by two vertical dashed lines.

Fig. 3 illustrates the behavior of the anode and cathode signals as recorded from a ${ }^{137} \mathrm{Cs} 662 \mathrm{keV}$ photo electrically absorbed event. The signals were sampled with 4 ns time period. However, for reasons of clarity the plots are made with the data re-binned into time channels of 16 ns duration.

The anode charge signals have a slow rise as long as the moving electron charge cloud is far away from the collecting anode strip and then finish with a rapid rise when the charge cloud is close the strip.

The total cathode charge signal, summed over the 10 cathode strips has an almost linear rise as long as the electrons drift through the detector volume. The cathode signal onset is marked with a red cross and defines the start time, $t_{s}$ of the electron drift.

The anode current displays a slow rise followed by a nearly Gaussian shape. In the data analysis we performed least square fits to the anode current signals assuming a linear dependence, using superimposed Gaussian functions. A typical result of the least square fitting is shown as the green curve in the lower left figure of Fig. 3. The center of the Gaussian function defines the time, $t_{c}$ where the charge cloud is fully collected by the anode. 

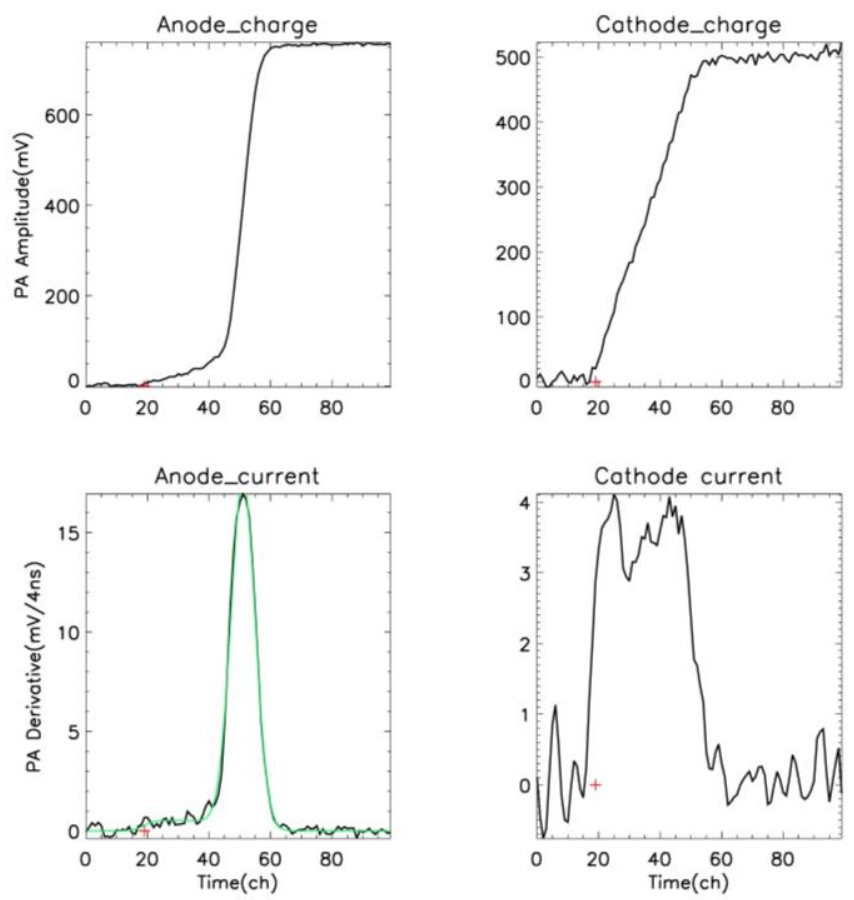

Fig. 3. Anode and cathode signal for $662 \mathrm{keV}$ Cs single interaction events. The upper plots display the anode and cathode charge signals and the lower curves show the current signals (i.e. the charge derivative in time). The time channels are in $16 \mathrm{~ns}$ bins. The red cross shows the start time $t_{s}$ of the charge collection.

\section{B. Double interaction events}

The event shown in Fig. 3 was assumed to originate from a single photon interaction within the drift cell. Double or multiple interaction events within one cell are, however frequent at photon energies above $300 \mathrm{keV}$. This is illustrated in Fig. 4 which shows the anode signal for a Compton scatter event, and subsequent absorption of the scattered photon in detector cell \#2. The two jumps in the anode charge signals corresponds to the collection of the two charge clouds created in two different locations in the cell. This is also reflected in the current signals shown in the lower left plot of Fig. 4. It clearly shows two separated peaks which can be fitted in the same manner as described in section A above.

The right hand plots display the signals obtained from the cathode plane. The cathode charge signal (upper) displays a slope change at the moment when the charge cloud closest to the anode plane is collected. The change in slope can also be seen in the cathode current signal displayed in the lower right plot of Fig. 4. Unfortunately, the cathode current signal suffers from a high noise level.

The time information is used to extract both energy and position for the Compton interactions and the scattered photon corresponding to the two peaks. These capabilities are very important for a spectral-imaging detector which can serve for astrophysical studies in the hard X-ray and/or soft gamma-ray band where Compton scattering processes can play an important role for photon detection.
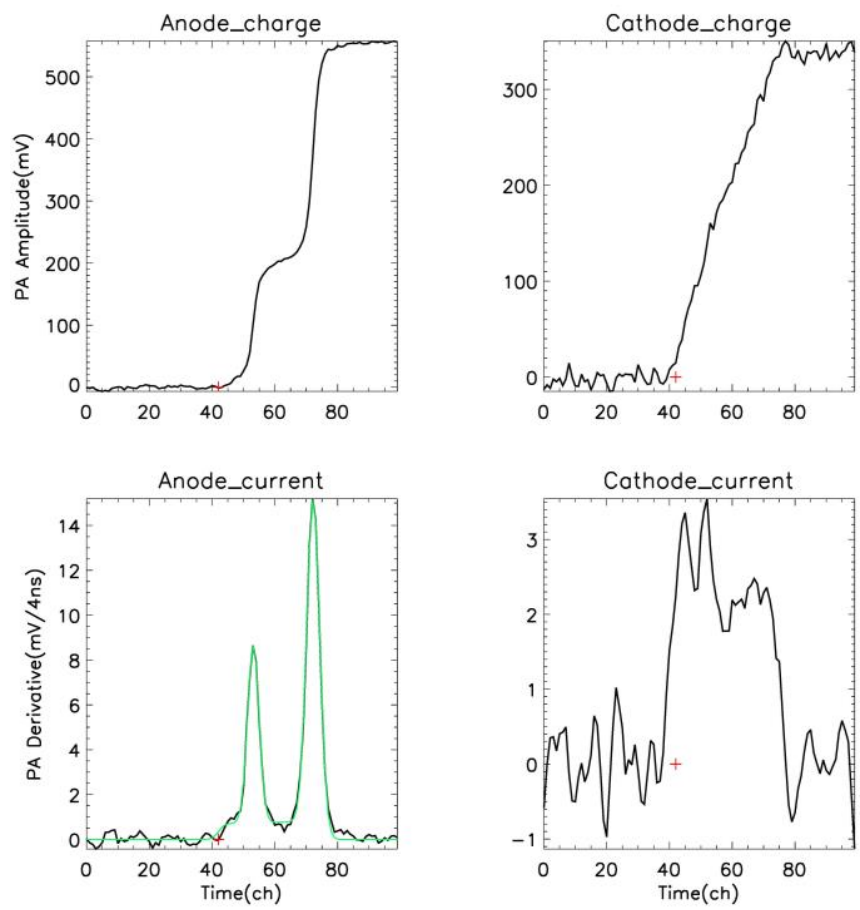

Fig. 4. Anode signals for double interaction event. The upper left plot displays the anode charge signal for a $662 \mathrm{keV}{ }^{137} \mathrm{Cs}$ double event. The anode current signal for this event is shown in the lower right plot. The cathode charge and current signals are shown in the right hand upper and lower figures respectively.

In the present study, however, we aimed to characterize the the 3D CZT detector system with respect to single photon interactions, so as to understand the optimal position and energy resolutions achievable with this system. Therefore double or multiple interaction events as discussed above were left out of the analysis. Also, shared events which triggered more than one anode were rejected.

\section{POSITION DETERMINATION}

When a photon interacts within the CZT detector, all electrodes record time coincident signals sampled with a $4 \mathrm{~ns}$ period during the electron drift time. This data can be analyzed to get information regarding 3D position, energy and time for each event. The employed position algorithm and the achievable position resolutions are discussed in the following sections.

\section{A. X-position}

When an anode is triggered, the signal will provide the interaction position in the $\mathrm{X}$-direction from the triggered anode number, $X_{\text {trig. }}$. This is a crude position with an uncertainty given by the anode strip pitch, $\mathrm{P}_{\mathrm{an}}=1.6 \mathrm{~mm}$. However, by utilizing drift strip signals (DL, DR) on the left and right side of the triggered anode, one can obtain a more precise position. An example of the charge and current signals from the left and right drift strip for, a single interaction, can be seen in Fig. 5.

The charge signals display an almost linear increase as the electron cloud drifts towards the drift strips and then a sudden turnover when it is collected at the anode strip. The (inverted) current signal was least square fitted to a linear function 
superimposed with a Gaussian peak at the collections time $t_{c}$ and with peak values of $A_{D L}$ and $A_{D R}$ for the left and right group, respectively. The least-square fits are displayed as green curves in Fig. 5. The interpolated $\mathrm{X}$-position, $\mathrm{X}_{\mathrm{pos}}$ is now calculated from:

$X_{\text {pos }}=\left[X_{\text {trig }}+F \cdot(-1)^{X_{\text {trig }}} \cdot \frac{\left(A_{D R}-A_{D L}\right)}{\left(A_{D R}+A_{D L}\right)}\right] \cdot P_{a n}$

Where $\mathrm{P}_{\mathrm{an}}$ is the anode strip pitch, and $\mathrm{F}$ is an adjustable factor required for the calibration of the $\mathrm{X}$ scale. For an ideal position response $\mathrm{F}=0.5$, however charges close to the cell borders will produce amplitudes in both DR and DL, so that $\mathrm{F}$ $>0.5$. For the present detector we find $\mathrm{F}=0.69$.
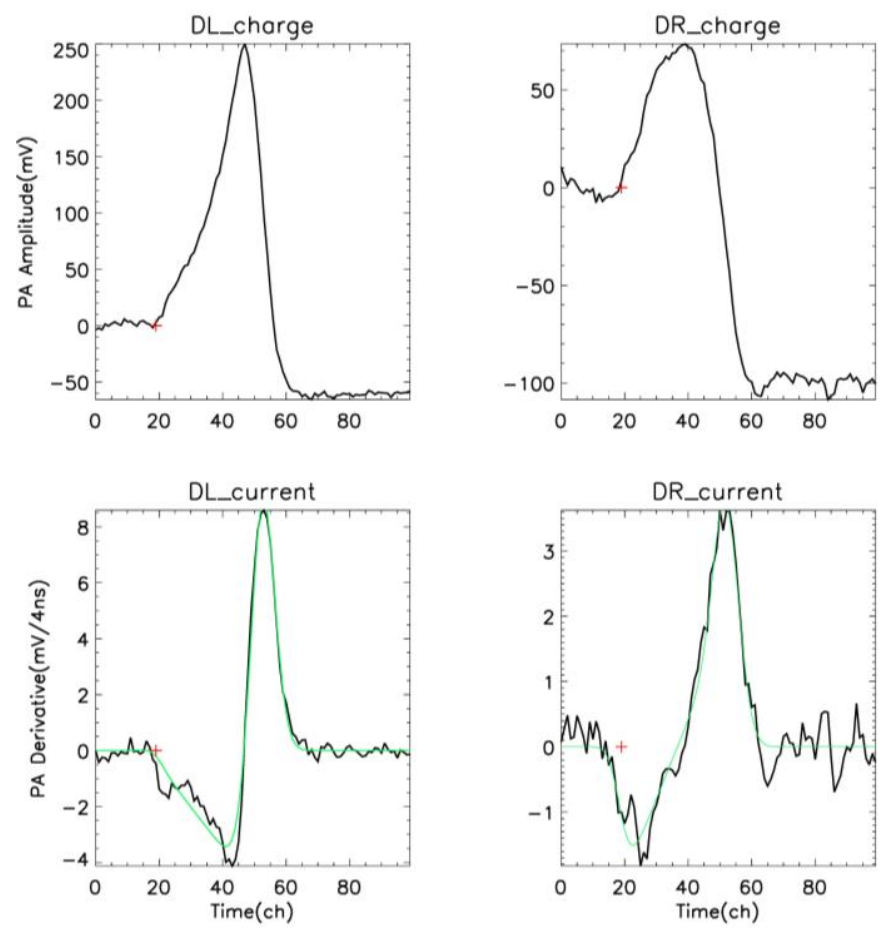

Fig. 5. Typical drift strip signals. Left: charge and current (inverted) signals from the left drift strip group (DL). Right: charge and current (inverted) signals from the right drift strip group (DR).

Notice that $A_{D L}$ for the event in Fig. 5 is more than a factor of 2 larger than $A_{D R}$, which means that the event was created to the left of the readout anode.

The detector was scanned in the X-direction using the 200 $\mu \mathrm{m}$ wide $662 \mathrm{keV}$ beam, in steps of $400 \mu \mathrm{m}$. The position response is shown in Fig. 6. The overall X-position resolution is $410 \mu \mathrm{m}$ (FWHM). Photons absorbed close to the borders between two detector cells will result in charge-shared events with simultaneous signals on two adjacent anodes. As stated previously, these events are rejected in the present configuration, which results in the drop in count rate at the border $(\sim 4 \mathrm{~mm})$ between cells number 2 and 3 . The rejection of charge-shared events can also be seen in the sine wave form of the background level in Fig. 6. Fig. 7 displays an image of the slit in the $\mathrm{X}-\mathrm{Y}$ plane. It is gratifying that the $\mathrm{X}$ - position determination is almost independent of the $\mathrm{Y}$ - position.

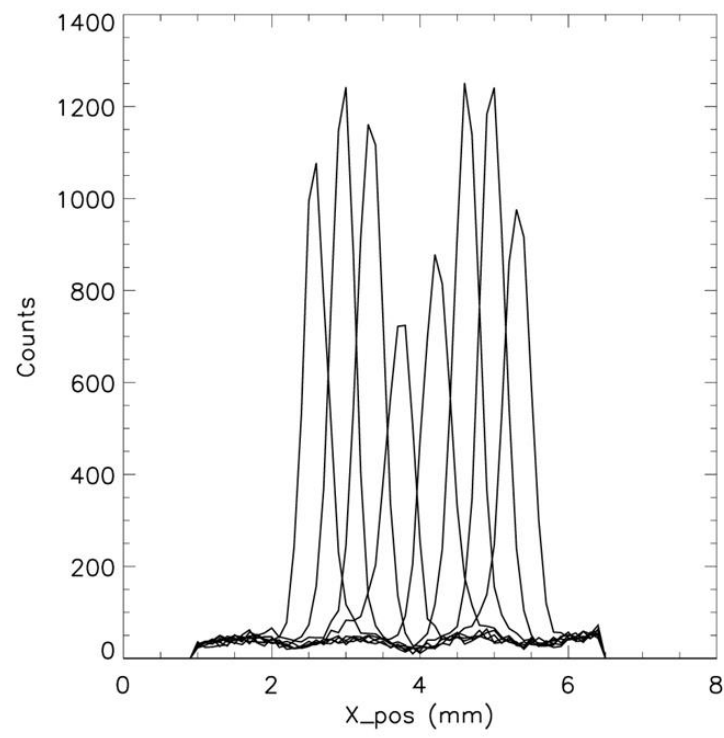

Fig. 6. X-position scans. The detector was moved in steps of $400 \mu \mathrm{m}$ in front of the $200 \mu \mathrm{m}$ opening of the $\mathrm{W}$ collimator slit.

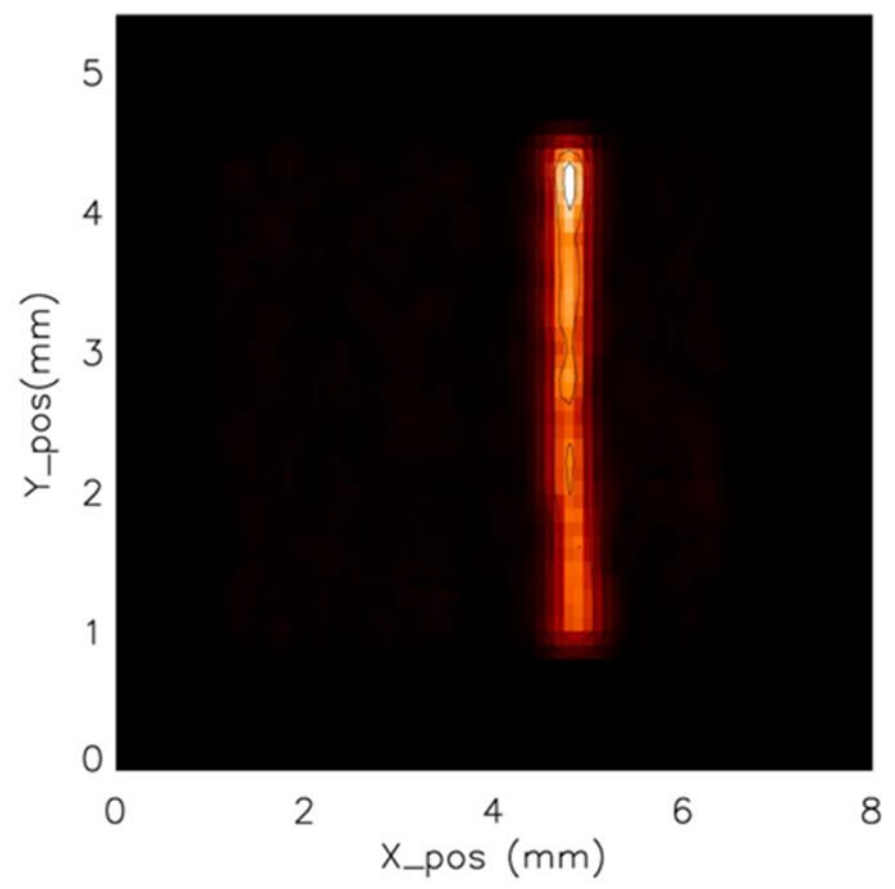

Fig. 7. Image of the $\mathrm{W}$ slit recorded in the detector $\mathrm{X}-\mathrm{Y}$ plane.

\section{B. Y-position}

The detector Y-positions were determined using the so called Depth of Interaction (DOI) method [3]. The ratio between the total cathode charge signal, $\mathrm{Q}_{\text {cathode }}$ and that of the collecting strip anode, $Q_{\text {anode }}$ is according to this method proportional to the Y-position:

$$
Y_{\text {pos }} \propto Q_{\text {anode }} / Q_{\text {anode }}
$$

This relation was checked in great detail in [2] employing finely collimated beams at ESRF in the energy range from 150 to $700 \mathrm{keV}$. The method was also tested with the present 662 $\mathrm{keV}$ slit beam. The results are shown in Fig. 8. The detector was scanned in the Y-direction with the $200 \mu \mathrm{m}$ wide $662 \mathrm{keV}$ beam in steps of $400 \mu \mathrm{m}$. The overall Y-position resolution is 
$420 \mu \mathrm{m}$ (FWHM).

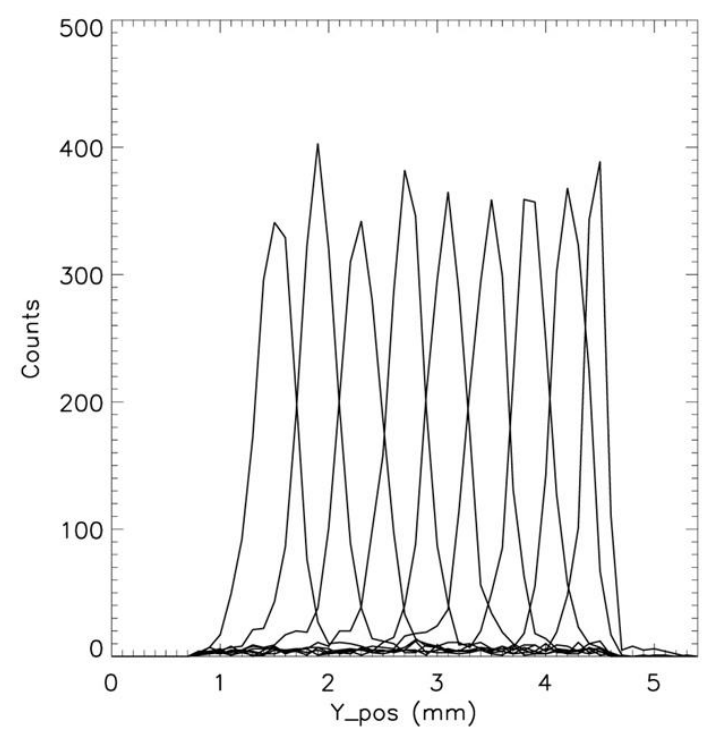

Fig. 8. Y-position scans. The detector was moved in steps of $400 \mu \mathrm{m}$ in front of the $200 \mu \mathrm{m}$ opening of the $\mathrm{W}$ collimator slit.

\section{Z-position}

The Z-position of the photon interaction is determined from charges, $\mathrm{Q}_{\mathrm{ci}}(\mathrm{i}=1$ to 10$)$ induced in the 10 cathode strips as the electron cloud drifts and is collected at the anode strips. See Fig. 9.

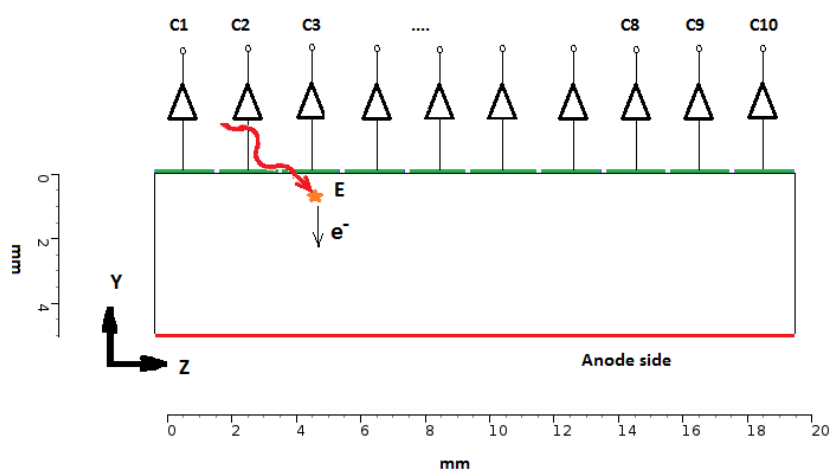

Fig. 9. Layout of the detector cathode strips used for determination of the Zposition. The cathode strip pitch is $\mathrm{P}_{\mathrm{ca}}=2.0 \mathrm{~mm}$ and the strip width is $\mathrm{W}_{\mathrm{ca}}=1.9$ $\mathrm{mm}$.

The Z-position is given by the center of gravity of the cathode strip charges in a first approximation:

$$
Z_{\text {pos }}=\left[\frac{\sum i \cdot Q_{c i}}{\sum Q_{c i}}\right] \cdot P_{c a}
$$

This relation was also checked in [2] with the help of the hard X-ray beam at ESRF. In general positions derived by (3) are accurate. However, (3) has a serious short-coming for events where absorption takes place close to, or just below a cathode strip. The movement of the electron cloud in such cases will result in induced charges on the cathode strips, which are equal to the cloud charge for the cathode strip in question and vanishing for all other strips. But the induced charge distribution is independent of the fine start position of the cloud on the strip. This can be verified e.g. by the
Shockley-Ramon Theorem [4], for example, applied to actual weighting potentials for the cathode strips. The consequence is that for near cathode strip events position determinations based on (3) will degenerate to the cathode strip positions and therefore have a limited accuracy of $\pm \mathrm{P}_{c a} / 2$. The time digitized cathode signals offer an elegant way of circumventing this problem

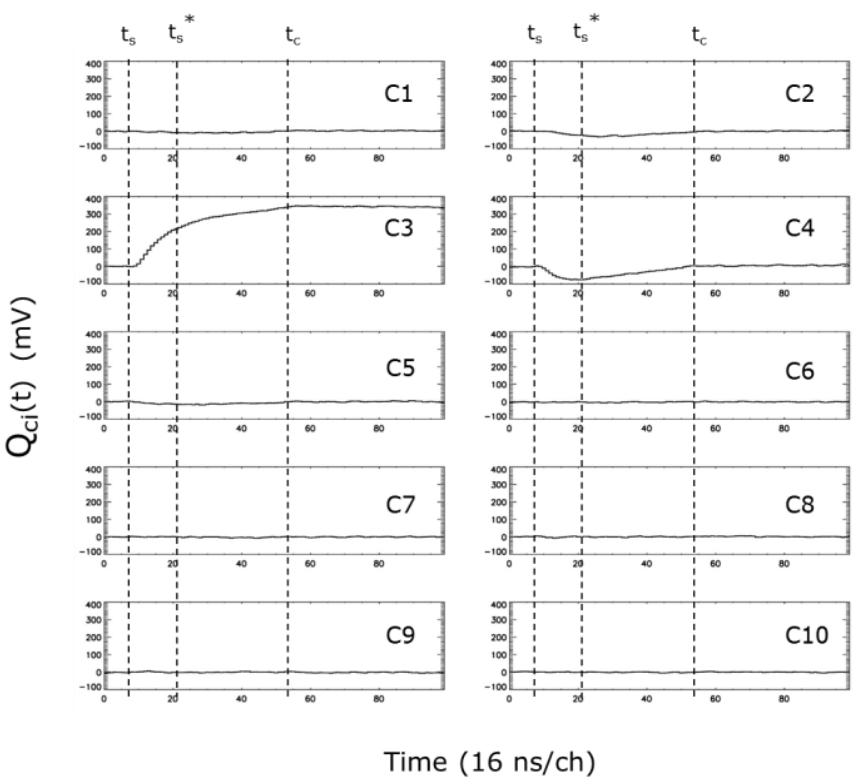

Fig. 10. The time dependence of the 10 cathode strip signals for a photon interaction event close to cathode strip \#3.

The event shown in Fig. 10 occurred close to strip\#3 which is the only strip with a non-vanishing charge after the electron charge collection. Also shown are the start time, $t_{s}$ and time of collection, $t_{c}$ for all strips. The electron drift time, $t_{c}-t_{s}$ for this event is close to the cathode-to-anode drift time confirming that the electrons for this event start drifting close to the cathode plane. The charge signal on strip\#3 starts out with a steep slope which decreases until full collection. The signals on the neighboring strips $(\# 2, \# 4)$ on the other hand, start out with negative slopes which after some time become positive such that the total induced charge signals at $t_{c}$ are very close to zero. This behavior of the cathode strip signals can be explained with the help of the Shockley-Ramon Theorem [4]. The position derived for this event using (3) will obviously be $3^{*} \mathrm{P}_{\mathrm{ca}}$; albeit with an uncertainty of $\pm \mathrm{P}_{\mathrm{ca}} / 2$. However, position accuracies better than the strip pitch can be obtained if (3) is used not with charge induced over the full drift time, $\mathbf{d t}=\mathbf{t}_{\mathbf{c}}-\mathbf{t}_{\mathbf{s}}$, but with the charge induced over the last part of the collection:

$$
Q_{c i}^{*}=Q_{c i}^{*}\left(t_{c}\right)-Q_{c i}^{*}\left(t_{s}^{*}\right)
$$

where $t_{s}{ }^{*}>t_{s}$ as illustrated in Fig. 10. The delayed start time $t_{s}{ }^{*}$ for this analysis is chosen by the algorithm below:

$$
\begin{aligned}
& t_{s^{*}}=t_{c}-d^{*} \text { for } d^{*}>d^{*} \\
& t_{s}=t_{s} \text { for } d t \leq d t
\end{aligned}
$$

Where $\mathbf{d t}^{*}$ is an adjustable drift time parameter. The actual value for $\mathbf{d t}^{*}=640 \mathrm{~ns}$, used in Fig.10, was chosen empirically. 
However, we are developing a simulation tool at DTU in order to calculate the optimal value for $\mathbf{d t}^{*}$.

Equation (4) ensures that the induced strip charges, $\mathbf{Q}^{*}{ }_{\mathrm{ci}}$ take values which the electron cloud would induce starting closer to the anode.

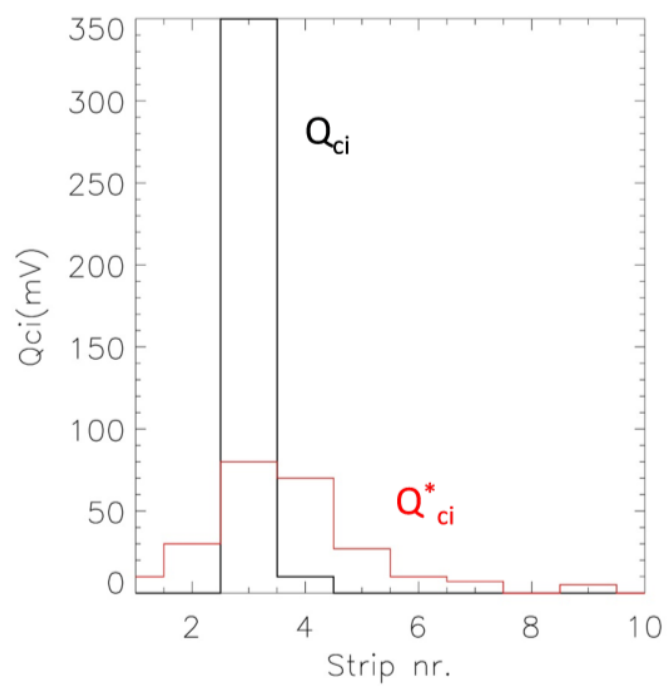

Fig. 11. The cathode strip charge distribution for an event close to cathode strip \#3. $\mathbf{Q}_{\mathrm{ci}}$ (black) denotes the fully integrated charge distribution whereas Q* ${ }_{\text {ci }}$ (red) denotes the distribution calculated from (4).

The difference between the two sets of induced signals: $\mathbf{Q}_{\mathbf{c}}$ and $\mathbf{Q}^{*}{ }_{\mathrm{ci}}$ are shown in Fig. 11. Remark that $\mathbf{Q}_{\mathrm{ci}}$ is concentrated on one cathode strip, \#3 whereas $\mathbf{Q}^{*}{ }_{\mathrm{ci}}$ is distributed over several strips yielding centroid determinations (3) with subpitch accuracy.

The above technique was tested using the $662 \mathrm{keV}$ slit beam. The left plot of Fig. 12 displays the position response obtained by illuminating the detector with $662 \mathrm{keV}$ photons through the $200 \mu \mathrm{m} \mathrm{W}$ slit moved in steps of $400 \mu \mathrm{m}$ along the Z-axis. The $\mathrm{Z}$ position resolution from these scans was determined to be $380 \mu \mathrm{m}$. The right figure shows 4 images of the slit in the $\mathrm{y}-\mathrm{z}$ plane each translated by $400 \mu \mathrm{m}$. The position response is preserved even for events with Y-positions close to the cathode plane at $4.5 \mathrm{~mm}$. The advantage of the presented technique is further illustrated in Fig. 13, which displays $\mathrm{Y}, \mathrm{Z}$ flat field images obtained by uniform illumination of a part of the detector. The left hand image was obtained using $\mathrm{Z}$ positions calculated according to (3), and the Z-position degeneration for events close to the cathode plane is clearly visible. The right hand image used $\mathrm{Z}$ positions calculated using the $\mathbf{Q}^{*}{ }_{\text {i }}$ values of (4): Here the degeneration has been lifted and the image reproduces the uniform illumination to a much high degree. The residual non-uniformity which still can be seen close to the cathode plane will be analysed further when our simulation tool is available.
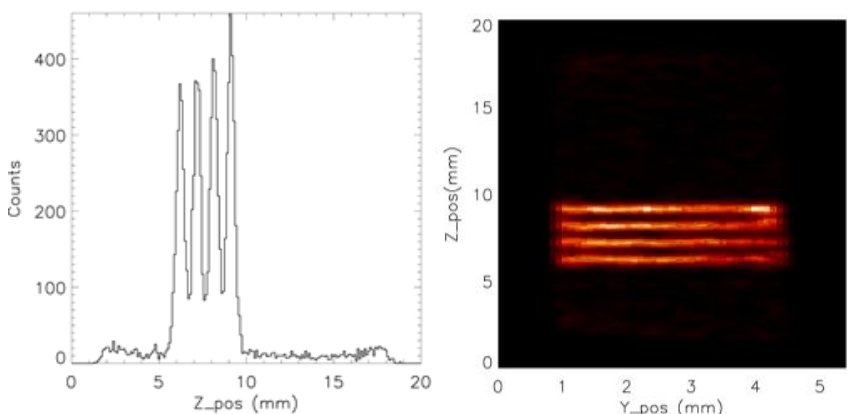

Fig. 12. Z-positions are determined using the signals induced on the 10 cathode strips. The left plot displays the position response obtained by illuminating the detector with $662 \mathrm{keV}$ photons through a $200 \mu \mathrm{m} \mathrm{W}$ slit moved in steps of $400 \mu \mathrm{m}$ along the Z-axis. The right hand figure shows 4 images of the slit in the $y-z$ plane each translated by $400 \mu \mathrm{m}$.
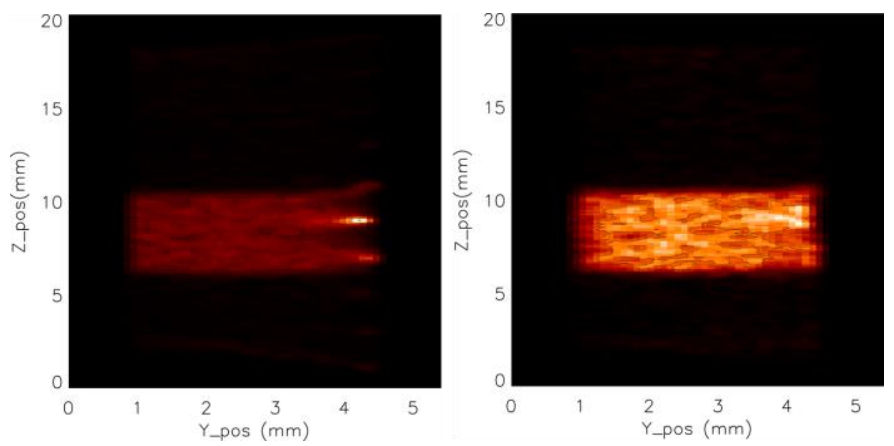

Fig.13. YZ flat-field images obtained by uniform illumination of a part of the detector. The left hand image was obtained using Z-positions calculated according to equation (3), whereas the right hand image used Z-positions calculated using the $\mathbf{Q}^{*}$ ci values of equation (4).

The measured position resolutions of the 3D CZT detector at $662 \mathrm{keV}$ are similar: 400 $\pm 30 \mu \mathrm{m}$ in all 3 (X,Y,Z) directions. The finite beam size of $225 \mu \mathrm{m}$ on the detector, contributes to this value. The resolution is further degraded by the variance of centroid of the charge distribution generated by photoelectrons. We estimated with the help of the monte-carlo simulation tool, CASINO [7] that the photoelectron contribution to the resolution is $250 \mu \mathrm{m}$ for the $662 \mathrm{keV}$ line. Thus these two components, which summed quadratically to $336 \mu \mathrm{m}$, account for the major part of the measured position resolution.

\section{ENERGY DETERMINATION}

An important advantage of a 3D position sensitive detector is that it makes it possible to determine and correct for position dependent pulse height variations throughout the detector volume and thereby to improve the overall energy resolution. Below we will discuss the energy algorithm developed for the present detector. The analysis is based on a data set obtained by full illumination of the detector $\mathrm{YZ}$ plane by the ${ }^{137} \mathrm{Cs}$ source. The aim of the present analysis was to map in 3D the detector response to $662 \mathrm{keV}$ photo-electrically absorbed photons.

The dominant position dependence of the pulse height is given by its $\mathrm{Y}$ position dependence. Two opposing effects are at play: 
1. The anode weighting potential for the drift strip detector results in induced signals which are reduced close to the anode (small Y position), [3].

2. Electron-trapping leads to reduced pulse heights for the largest drift distances (large Y position). However, the high quality $\left(\mu \tau_{\mathrm{e}}\right.$ of $\sim 0.01 \mathrm{~cm}^{2} / \mathrm{V}$ ) of the employed CZT material ensures that trapping loses are rather small $(<5 \%)$.

The above effects are well understood and discussed in [3] for example. Fig. 14 displays the pulse height of $662 \mathrm{keV}$ photons detected by photoelectric absorption as a function of their Yposition. The pulse height decreases by $10 \%$ going from the cathode side at $4.5 \mathrm{~mm}$ to $0.9 \mathrm{~mm}$ above the anode plane. The pulse height decreases rapidly for smaller Y_position values closer to the anodes, and the width of the $662 \mathrm{keV}$ peak broadens. We therefore decided to reject these events (Y_pos $<0.9 \mathrm{~mm}$ ) in the present analysis.

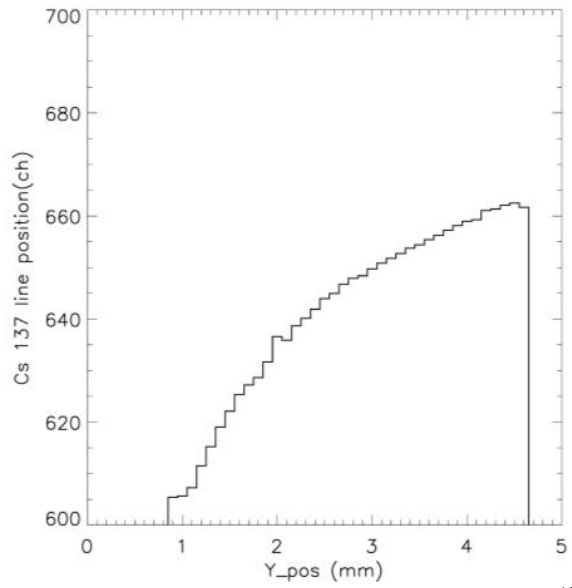

Fig. 14. Pulse height dependence of the Y-position for ${ }^{137} \mathrm{Cs} 662 \mathrm{keV}$ photons, detected by photoelectric absorption.

The anode strip pulse heights were as the first step corrected for the Y-position dependence depicted in Fig. 14. This reduced the FWHM of the integrated $662 \mathrm{keV}$ peak from $4.6 \%$ to $1.7 \%$. The resulting pulse heights were then investigated with respect to residual dependence on the X-position and Zposition. Fig. 15 displays the pulse height dependence as function of the X-position integrated over all events. It shows sinusoidal behavior with maxima at the drift strip anodes. The rightmost data show a somewhat different behavior since they belong to an edge drift cell which has only one neighboring drift cell. Actually, this corresponds to the first drift cell shown in Fig 1.

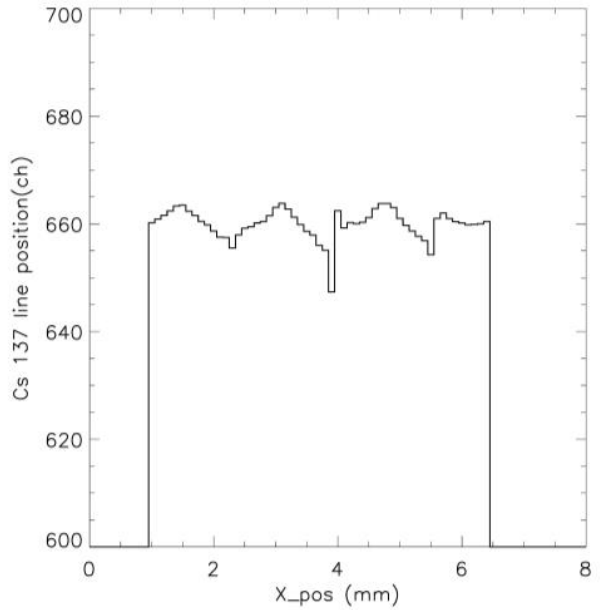

Fig. 15. Pulse height dependence on the X-position for ${ }^{137} \mathrm{Cs} 662 \mathrm{keV}$ photons, detected by photoelectric absorption.

Fig.16 displays the pulse height dependence as a function of the Z-position integrated over all events. The 1-2\% variations are probably caused by CZT material inhomogeneity effects. Similarly sized variations might be hidden in the more pronounced behavior of the $\mathrm{X} / \mathrm{Y}$-position pulse height dependencies.

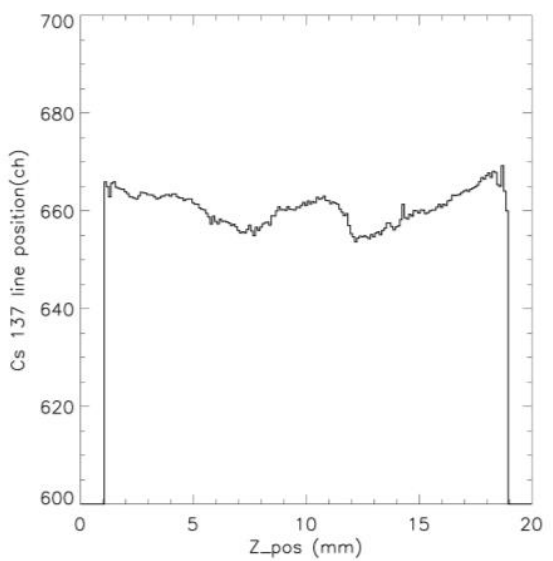

Fig. 16. Pulse height dependence on the $\mathrm{Z}$ position for ${ }^{137} \mathrm{Cs} 662 \mathrm{keV}$ photons detected, by photoelectric absorption.

The pulse heights were corrected for these $\mathrm{X} / \mathrm{Z}$ - position dependent variations by forming a $2 \mathrm{D}$ correction matrix by calculating the mean pulse height of the $662 \mathrm{keV}$ line in pixels of $\Delta X=0.5 \mathrm{~mm}$ and $\Delta \mathrm{Z}=1.0 \mathrm{~mm}$. Application of the $\mathrm{X} / \mathrm{Z}$ correction to the whole data-set resulted in the spectrum shown in Fig. 17. The combined X/Z correction improved the energy resolution further from $1.7 \%$ to $1.14 \%$. 


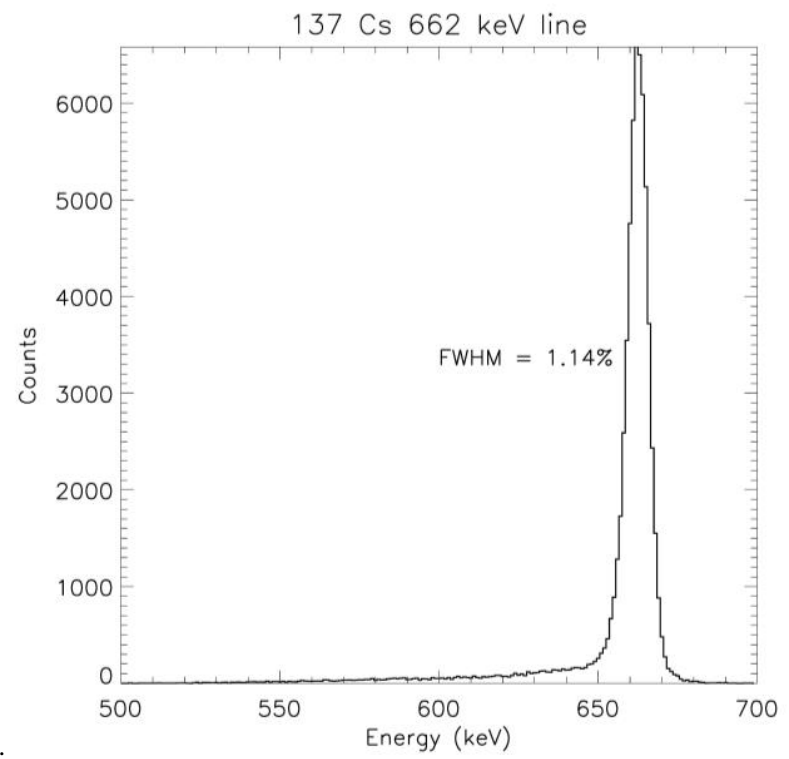

Fig.17. The resulting pulse height distribution of ${ }^{137} \mathrm{Cs} 662 \mathrm{keV}$ photoelectrically absorbed photons after $\mathrm{Y}$ correction (1D) and subsequent $\mathrm{X}, \mathrm{Z}$ correction (2D).

\section{CONCLUSION}

The 3D position sensitive CZT detector for high energy astrophysics developed at DTU has been investigated with a digitizer readout system.

The 3D CZT setup was characterized using a fine collimated, high-energy monochromatic beam at DTU Space. With the developed position algorithms, presented here, we demonstrate improved position determination compared to the previous read-out system based on analog electronics. The strong correlation between measured $\mathrm{X}$ and Y-positions observed previously [2] is now resolved. For the Z-position the technique presented here preserves the high sub-pitch resolutions even for events absorbed close to the cathode plane. Overall position resolutions of $0.4 \mathrm{~mm}$ FWHM in the $\mathrm{X}, \mathrm{Y}$ and Z-directions were achieved at $662 \mathrm{keV}$. Furthermore, the fine 3D position information allowed detailed corrections for position-dependent pulse height effects. Hereby the obtainable energy resolution for the fully illuminated detector was improved to $\sim 7.5 \mathrm{keV}$ FWHM at $662 \mathrm{keV}$.

The timing information also makes it possible to identify of Compton events. These characteristics are very desirable for a spectral-imager serving as instrumentation for astrophysical studies in the hard X-ray and/or soft gamma-ray band. Future investigations of the 3D CZT detector will be devoted to its performance in a broader energy band as well as development of the treatment of double or multiple interaction events. Preferably these investigations and tests will repeat of the former test campaign [2] at ESRF.

\section{REFERENCES}

[1] I. Kuvvetli, C. Budtz-Jørgensen, "Charge Collection and Depth Sensing Investigation on CZT Drift Strip Detectors," IEEE Nuclear Science Symposium Conference Record NSS '11 (R7-2), 2010
[2] I. Kuvvetli, C. Budtz-Jørgensen, et al., ”A 3D CZT high resolution detector for X-and gamma-ray astronomy," Proceedings of SPIE, Vol. 9154, 2014, DOI: http://dx.doi.org/10.1117/12.2055119

[3] M.A.J. van Pamelen and C. Budtz-Jørgensen, "CdZnTe drift detector with correction for hole trapping", Nucl. Instr. and Meth., A 411, pp 197-200, 1998.

[4] W. Shockley "Currents to conductors induced by a moving point charge" J. Appl. Phys.,vol. 9, p. 635, 1938.

[5] D. Della Monica Ferreira, F. E. Christensen, M. J. Pivovaroff, N. F. Brejnholt, M. Fernandez-Perea, N. J. S.Westergaard, A. C. Jakobsen, M.-A. Descalle, and R. Soufli, "Hard x-ray/soft gamma-ray telescope designs for future astrophysics missions," Proceedings of SPIE, Vol. 8861, pp. 886116, 2013.

[6] E. Caroli, et al., "A 3D CZT hard x-ray polarimeter for a balloon-borne payload," Proceedings of SPIE, Vol. 8443, p844340, 2012

[7] Alexandre Réal Couture et. al., CASINO, monte CArlo SImulation of electroN trajectory in sOlids, v2.48 (2.4.8.1), "http://www.gel.usherbrooke.ca/casino/index.html", 2011 